\title{
Evaluation of a new system (RAD I 20) for the serologic determination of the T.O.R.C.H complex analytes
}

Mauro Carcheri, Ilaria Chiossone, Paola Milano, Anna Alabiso, Fabrizia Capanna, Annamaria Ferretti,
Licia Muselli, Caterina Oliveri, Laura Tassi, Roberto Capuzzo
ASL 3 Genovese - Dipartimento di Patologia Clinica - U.O. Laboratorio Analisi Chimico-Cliniche e Microbiologiche - Presidio Ospedaliero Metropolitano "Villa Scassi", Genova.

Key words: Antigen, Antibody, IgG, IgM, T.O.R.C.H. complex

Valutazione di un nuovo sistema (RAD I20) per il dosaggio sierologico degli analiti del complesso T.O.R.C.H.

SUMMARY

The aim of this study was to evaluate the results of the determination of $\lg G$ and $\lg M$ against TORCH complex antigens obtained by using a new automatic random-access (RAD I20 - RADIM/SEAC) instrument based on FEIA method, in comparison with the results obtained for the same analytes by using a traditional analyzer based on a coated microplate ELISA method (ALISEI-RADIM/SEAC). The analysis of the discordances between the results was made with the system ELFA (VIDAS - bioMérieux), taken as a reference test.

The general concordance of the results obtained with the FEIA and the ELISA was over $97.00 \%$. For the single analyte as Toxo IgG, Toxo IgM, Rubeo IgM, CMV IgG, CMV IgM and HSV 2 lgG, the concordance was above $96.00 \%$ and $100.00 \%$ for the Rubeo $\lg$ and HSV I IgG. For the HSV I/2 IgM, the FEIA/ELISA results show lower concordance, but still reaching $90.16 \%$.

For the IgG and IgM dosage, the FEIA seems to give more positive results than the ELISA. If the discordances FEIA/ELISA concern the IgG, the ELISA seems to agree better with the ELFA results. For the IgM dosage, all the FEIA positive/ELISA negative results have been confirmed as negative at ELFA. In conclusion the new technology FEIA/RADI20 gave results which can be comparable with those obtained at the traditional ELISA as well at the alternative ELFA.

\section{INTRODUZIONE}

Le infezioni del complesso TORCH possono risultare pericolose per il feto soprattutto se contratte nelle prime fasi del suo sviluppo e il rischio, oltre a variare a seconda dell'agente patogeno responsabile, diminuisce con il progredire della gravidanza (6). Inoltre queste infezioni non sempre esitano in una forma clinica, soprattutto in presenza di normali difese immunitarie. Perciò, prima di iniziare una gravidanza, sarebbe utile fosse effettuato sulla candidata mamma un controllo dello stato immunitario nei confronti degli agenti del complesso TORCH per sapere se è protetta o no nei confronti di queste infezioni. In presenza IgG specifiche non sono necessari altri controlli durante la gravidanza. L'assenza di IgG specifiche indica invece la recettività della donna in esame ad una possibile futura infezione e, in corso di gravidanza, impone una serie di misure preventive atte a impedire, o rendere meno probabile, il contrarre un'infezione primaria, nonché il monitoraggio anticorpale mensile, per poter individuare precocemente un'eventuale infezione primaria $(1,10)$. Inoltre è noto che, pur con tutti i limiti descritti in letteratura (2), le IgM possono essere indice di infezione in atto o recente e, pertanto, il loro riscontro può dare importanti indicazioni sullo stato dell'eventuale infezione e sui possibili rischi fetali (9). Allo stato attuale per la ricerca di IgG e IgM per gli antigeni del complesso TORCH si utilizzano metodiche immunoenzimatiche in micropiastra (ELISA), metodiche in microstrip (ELFA) o metodiche in chemiluminescenza (FEIA, CLIA, MEIA) (3 - 5). In ogni caso, visto il carattere di routine dei test in oggetto e la necessità di avere risultati attendibili e in tempi veloci, sono stati sempre più privilegiati sistemi automatici in grado di processare anche notevoli quantità di analiti, rapidamente e con risultati standardizzabili . Obiettivo di questo studio è stata la valutazione dei risultati ottenuti dalla determinazione delle IgG/IgM per gli antigeni del complesso TORCH ottenuta utilizzando un nuovo strumento automatizzato random-access (RAD 120 RADIM/SEAC) che utilizza la metodica FEIA, in comparazione con i risultati ottenuti per gli stessi analiti utilizzando un classico analizzatore che utilizza la metodica ELISA in micropiastra (ALISEI - RADIM/SEAC) (8). L'analisi delle discordanze tra i risultati ottenuti con le due metodiche è stata effettuata con il sistema ELFA (VIDAS - bioMérieux) (11), preso come test di riferimento.

\section{MATERIALI E METODI}

Sono stati testati, in totale, 2737 analiti: Toxoplasma gondii (Toxo) IgG 745 campioni, Toxo IgM 260, Rubella virus (Rubeo) IgG 244, Rubeo IgM 248, CMV IgG 248, CMV IgM 288, HSV 1 IgG 212, HSV 2 IgG 244, HSV M 220.

I campioni provenivano per la quasi totalità da pazienti di sesso femminile in monitoraggio per gravidanza (esami pre-concezionali, check up mensile di soggetti recettivi in corso di gravidanza). Non ci sono state richieste di determinazione dello stato anticorpale per una sospetta infezione recente o in atto.

Per la ricerca delle IgG, i sieri in esame sono stati processati su analizzatore automatico ALISEI (RADIM SpA - SEAC) utilizzando una metodica immunoenzimatica (ELISA) in micropiastre da 96 pozzetti nei quali è adeso l'antigene (Toxoplasma, Rosolia, CMV, HSV-1, HSV-2 IgG/IgM EIA WELL - RADIM) e su analizzatore RAD 120 - Analizzatore ad Accesso Random, caricamento continuo, utilizzando una metodica FEIA che impiega un Sistema Immunometrico Enzimatico in Fluorescenza su Particelle Magnetiche (RAD Toxoplasma, Rosolia, CMV, HSV-1 e HSV-2 IgG e IgM RADIM). Per la ricerca delle IgM, i sieri in esame sono stati processati su analizzatore ALISEI utilizzando una metodica immunoenzimatica (ELISA) “a cattura”, anche questa basata sull'utilizzo di micropiastre da 96 pozzetti (Toxoplasma, Rosolia, CMV, IgM EIA WELL "cattura” - RADIM). Il dosaggio immunoenzimatico "a cattura” utilizza anticorpi anti catena $\mu$ delle IgM umane, adesi alla superficie dei pozzetti della micropiastra di reazione e solo in una seconda fase viene aggiunto l'antigene specifico per selezionare gli anticorpi specifici eventualmente "catturati”. La metodica ELISA impiegata per la ricerca delle IgM anti HSV è stata, invece, una tradizionale metodica "sandwich" che utilizza micropiastre a 96 pozzetti che recano adeso l'antigene HSV. La metodica FEIA IgG si basa sullo stesso principio della metodica ELISA ma impiega come fase solida particelle magnetiche di ferrite rivestite di zirconia. Queste, grazie alle loro caratteristiche chimicho-fisiche, hanno una elevata capacità legante verso molecole biologicamente attive e consentono una rapida e

\section{Corresponding author: Mauro Carcheri}

U.O. Laboratorio Analisi - Presidio Ospedaliero Metropolitano "Villa Scassi” - ASL 3 Genovese

16149 Genova - Corso O.Scassi, I - Tel. 010410239

E-mail: mauro.carcheri@villascassi.it 
sicura separazione della frazione libera da quella legata. La metodica è applicabile a protocolli analitici diretti, indiretti, a cattura. La lettura avviene in fluorescenza a $450 \mathrm{~nm}$ utilizzando come substrato 4-metilumbelliferil fosfato. Anche la metodica FEIA IgM è una metodica "a cattura” (ad esclusione del kit HSV IgM). Una terza metodica che utilizza la tecnologia ELFA a fluorescenza (Toxoplasma, Rosolia, CMV IgG e IgM VIDAS - bioMérieux) è stata infine utilizzata per saggiare i campioni che con le due metodiche a confronto avevano fornito risultati discordanti per uno o più analiti.

\section{RISULTATI}

I risultati dei due metodi (FEIA/RAD120 e ELISA/ALISEI) hanno concordato nella seguente misura: Toxo IgG 97.72\%, Toxo IgM 96.83\%, Rubeo IgG 100.00\%, Rubeo IgM 96.67\%, CMV IgG 96.78\%, CMV IgM 98.96\%, HSV1 IgG 100.00\%, HSV2 IgG 98.37\%, HSV 1+2 IgM 90.16\%. (Tabella 1).

Tabella I. Percentuali di concordanza ELISA/FEIA per $i$ vari analiti:

\begin{tabular}{lc}
\hline ANALITA & CONCORDANZE \\
\hline TOXO IgG & $\mathbf{9 7 . 7 2} \%$ \\
\hline TOXO IgM & $\mathbf{9 6 . 8 3} \%$ \\
\hline RUBEO IgG & $\mathbf{1 0 0 . 0 0 \%}$ \\
\hline RUBEO IgM & $\mathbf{9 6 . 6 7 \%}$ \\
\hline CMV IgG & $\mathbf{9 6 . 7 8 \%}$ \\
\hline CMV IgM & $\mathbf{9 8 . 9 6 \%}$ \\
\hline HSVI IgG & $\mathbf{1 0 0 . 0 0 \%}$ \\
\hline HSV2 lgG & $\mathbf{9 8 . 3 7 \%}$ \\
\hline HSV IgM & $\mathbf{9 0 . 1 6 \%}$ \\
\hline
\end{tabular}

Per quello che riguarda le discordanze, il raffronto tra metodica FEIA su strumento RAD120 e metodica ELISA su strumento ALISEI, per ogni analita, ha fornito i dati riportati in Tabella 2. I campioni che hanno dato risultati discordanti sono stati testati anche con il sistema ELFA/VIDAS (bioMérieux) con i seguenti risultati:

Tabella 2a

- Toxo lgG: 8 positivi FEIA/negativi ELISA $\rightarrow 8$ negativi ELFA. 9 negativi FEIA/positivi ELISA $\rightarrow 5$ positivi, 4 dubbi (risultato in gray-zone) ELFA.

- CMV IgG: 8 positivi FEIA/negativi ELISA $\rightarrow 6$ negativi, 2 dubbi (gray-zone) ELFA.

- Toxo IgM: 8 positivi FEIA/negativi ELISA $\rightarrow 8$ negativi ELFA. - Rubeo IgM: 8 positivi FEIA/negativi ELISA $\rightarrow 8$ negativi ELFA. - CMV IgM: 3 positivi FEIA/negativi ELISA $\rightarrow 3$ negativi ELFA

I risultati del test di conferma con la metodica ELFA sono riportati in Tabella 2.

La concordanza generale tra le metodiche ELISA/ALISEI e FEIA/RAD120 è risultata del 97.37\%, la discordanza del 2.63\%.

\section{CONCLUSIONI}

La concordanza generale dei risultati ottenuti con la metodica FEIA e con la metodica ELISA è risultata superiore al 97.00\%. Per i singoli analiti testati la concordanza risulta maggiore del 96.00\% (Toxo IgG, Toxo IgM, Rubeo IgM, CMV IgG, CMV IgM, HSV 2 IgG), con due casi di concordanza totale (100.00\%) nel caso di Rubeo IgG e HSV 1 IgG.
Nel caso del dosaggio HSV 1/2 IgM i risultati FEIA/ELISA concordano meno, ma comunque è raggiunta una concordanza del $90.00 \%$. Sia nel caso del dosaggio delle IgG specifiche che in quello del dosaggio delle IgM specifiche, la metodica FEIA sembra fornire più positivi della metodica ELISA. In particolare i risultati IgG positivi al test FEIA/negativi al test ELISA sono stati confermati come negativi con il test ELFA. Per l'analita Toxo IgG 9 campioni negativi al test FEIA e positivi al test ELISA, analizzati con il test ELFA sono risultati positivi in 5 casi e dubbi (risultato in gray-zone) in 4 casi. Nel caso delle discordanze FEIA/ELISA per quello che riguarda le IgG, la metodica ELISA sembra concordare meglio con i risultati forniti dal test ELFA, preso come test di riferimento.

Nel caso del dosaggio delle IgM, tutti i risultati positivi al test FEIA e negativi al test ELISA (8 Toxo IgM, 8 Rubeo IgM, 3 CMV IgM) sono stati confermati come negativi con il test ELFA, fatto che conferma la tendenza del test FEIA a dare una piccola percentuale di "falsi positivi" alla ricerca delle IgM. In conclusione è possibile affermare che la nuova tecnologia FEIA/RAD120 ha fornito risultati comparabili con quelli ottenuti con la tradizionale metodica ELISA in micropiastra e con la metodica alternativa in monostrip (ELFA/VIDAS), che può essere utilizzata quale test di conferma dei risultati IgM positivi o di quelli dubi. La modalità di accesso random dei campioni da processare, propria dell'analizzatore RAD 120 contribuisce, inoltre, a snellire il flusso di lavoro soprattutto nel caso di piccole serie di esami multipli.

\section{BIBLIOGRAFIA}

1. Bahar IH, Karaman M, Kirdar S, Yilmaz O, Celıloglu M, Mutlu D. The importance and validity of anti Toxoplasma gondii IgG, IgM, IgA antibodies and IgG avidity tests in the diagnosis of Toxoplasmosis infection during pregnancy.] Turkiye Parazitol Derg 2005; 29(2): 76-9.

2. De Paschale M, Agrappi C, Belvisi L, et al. Revision of the positive predictive value of IgM anti-Toxoplasma antibodies as an index of recent infection. New Microbiol 2008; 31(1): 105-11.

3. FDA, USA. NCCLS. Specifications for immunological testing for infectious diseases; Approved Guideline.NCCLS Document; 18(14): 1994 Dec. http://www.fda.gov

4. Li Jiang Zhangbin Yu, Weidong Du, Zuming Tang, Tao Jiang, Chunxiu Zhang, Zuhong Lu. Development of a fluorescent and colorimetric detection methods-based protein microarray for serodiagnosis of TORCH infections. Biosens Bioelectron 2008; 24(3): 376-82.

5. Maselli E, Capone G, Ricci M, Corio R. ToRCH IMMULITE $®$ : un nuovo sistema in chemiluminescenza per la determinazione del complesso ToRCH. Pandora, maggio-agosto 2000.

6. Remington JS, Klein JP. Infectious diseases of the fetus and newborn infant. Ed W.B. Saunders, Philadelphia, 1990, 89-195.

7. Sambri V, Simone MA, Cevenini R. Il Nuovo Sistema automatizzato per metodiche immunoenzimatiche in micropiastra ALISEI-RADIM, nella determinazione dello stato immune nei confronti degli agenti del complesso TORCH. Microbiol Med 2001; 16(2): 153-4.

8. Saracino M, Moroni A, Sambri V, Cevenini R. Valutazione delle performance analitiche dei test sierologici "a cattura" per la diagnosi delle infezioni acute da CMV, Toxoplasma gondii e Rubeovirus su sistema automatizzato per metodiche imunoenzimatiche in micropiastra ALISEI-RADIM. Microbiol Med 2002; 17(2): 252-3.

9. Turbadkar D, Mathur M, Rele M. Seroprevalence of torch infection in bad obstetric history.

10. Vlaspolder F, Singer P, Smit A, Diepersloot RJA. Comparison of IMMULITE® to VIDAS for antenatal screening in a low prevalence population in The Netherlands. Presented to ASM Meeting, July 2000.

Tabella 2. Analisi delle discordanze per tutti i risultati e analisi di conferma con la metodica ELFA

\begin{tabular}{|c|c|c|c|}
\hline & positivi FEIA/negativi ELISA & positivi ELISA/negativi FEIA & ELFA (vedi Tabella 2a) \\
\hline Toxo lgG & 8 su $745(1.07 \%)$ & 9 su $745(1.20 \%)$ & 8 negativi, 5 positivi, 4 dubbi \\
\hline Toxo IgM & 8 su $252(3.17)$ & 0 su 252 & 8 negativi \\
\hline Rubeo IgG & 0 su 244 & 0 su 244 & \\
\hline Rubeo IgM & 8 su $240(3.33 \%)$ & 0 su 240 & 8 negativi \\
\hline CMV IgG & 8 su $248(3.22 \%)$ & 0 su 248 & 6 negativi, 2 dubbi \\
\hline CMV IgM & 3 su $288(1.04 \%)$ & 0 su 288 & 3 negativi \\
\hline HSV I IgG & 0 su 212 & 0 su 212 & \\
\hline HSV $2 \operatorname{lgg}$ & 0 su 244 & 4 su $244(1.63 \%)$ & \\
\hline $\mathrm{HSV} \mathrm{I} / 2 \mathrm{lgM}$ & 8 su 244 (3.28\%) & 16 su $244(6.56 \%)$ & \\
\hline
\end{tabular}

